\title{
DISEÑO Y FABRICACIÓN DE UNA MÁQUINA PARA DESGRANAR MAÍZ
}

\section{DESIGN AND CONSTRUCTION OF A CORN SHELLER}

\author{
José Olger Pérez Silva, ${ }^{1, *}$ Luis Christian Cholca Cacuango², \\ Gustavo Andrés Mantilla Valencia ${ }^{2}$
}

\section{Resumen}

El presente artículo da a conocer el diseño y construcción de una desgranadora de maíz para sustituir el trabajo manual y monótono que causa lesiones y enfermedades en los agricultores. Esta tarea ocasiona afectaciones ocupacionales a la salud de la persona quien de forma artesanal desgrana 10 q de maíz diarios; entre las lesiones graves que pueden darse están la tendinitis en el dedo pulgar, lumbalgia en la espalda e hiperlordosis dorsal producida por una mala postura al desgranar. Ante esta situación se planteó una alternativa de trabajo al diseñar y posteriormente construir una máquina que realice el mismo trabajo remplazando el esfuerzo humano. Se describe el proceso de diseño y fabricación de la máquina, que permitió obtener una producción de 105 q al día de manera rápida y limpia, para desgranar todo tipo de maíz con una humedad del 10 al $15 \%$ además de abastecer la demanda en el mercado salvaguardando la salud física al evitar lesiones a las personas.

Palabras clave: enfermedad ocupacional, desgranadora, maíz, agroindustria, diseño de máquinas.

\section{Abstract}

This article lays out the design and construction of a corn sheller with the objective to replace the monotonous manual labor of those who shell corn, which often causes injuries and illnesses in farm workers. The arduous manual labor causes occupational damages to the health of the worker, who produce 10q of corn daily using the artisanal manner. Among the serious injuries which can arise are tendonitis of the thumb, lumbago of the back, and dorsal lordosis produced by shelling in a bad posture. Faced with this situation, an alternative was proposed: designing and subsequently constructing a machine which would carried out the same labor replacing human stress. The machine's design and construction process is described; which allowed obtaining $105 q$ daily in a fast and clean way, to thresh all kinds of corn with a humidity between 10 to $15 \%$, supplying the demand in the market and safeguarding the physical health of workers.

Keywords: Occupational disease, Sheller, Corn, Agroindustry, Machine design.

\footnotetext{
1,* Área de Mecánica y Mecatrónica, Colegio Técnico Salesiano Don Bosco - Ecuador, Autor para correspondencia : josuepepo@yahoo.com. (1) http://orcid.org/0000-0002-9991-3592

${ }^{2}$ Carrera de Ingeniería Mecánica, Universidad Politécnica Salesiana Sede Quito - Ecuador.

(D) http://orcid.org/0000-0001-6030-1241, (D) http://orcid.org/0000-0001-9391-129X
}

Recibido: 21-12-2016, aprobado tras revisión: 08-05-2017

Forma sugerida de citación: Pérez, J.; Cholca, L.; Mantilla, G. (2017). «Diseño y fabricación de una máquina para desgranar maíz». INGENIUS. N. ${ }^{\circ} 18$, (julio-diciembre). pp. 21-29. ISSN: 1390-650X. 


\section{Introducción}

El maíz es el cereal del continente americano y uno de los productos de mayor importancia mundial. En el Ecuador se siembran varios tipos de este grano; pero el maíz duro seco es el de mayor producción por el alto consumo tanto en la alimentación humana como para la elaboración de balanceado para consumo animal.

En la temporada de invierno del 2015 el rendimiento de maíz fue de 5,41 ton/ha tal como se muestra en la Figura 1. Las provincias de Los Ríos, El Oro y Loja fueron las de mayor producción en el Ecuador [1].

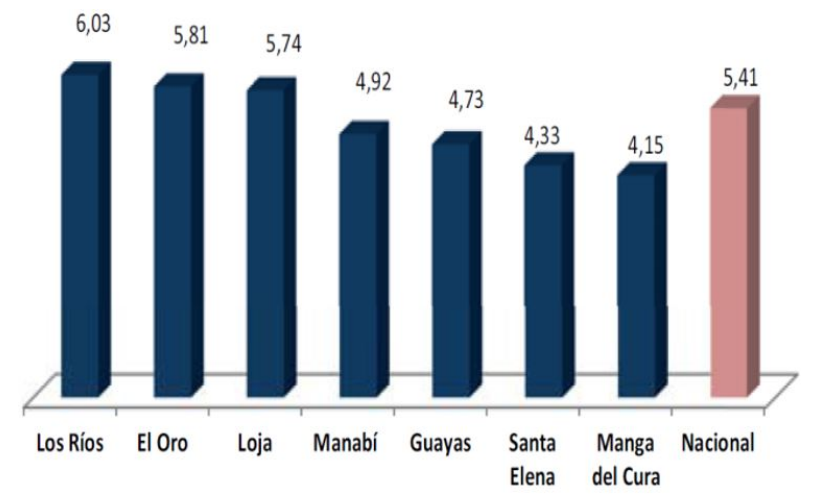

Figura 1. Rendimiento de maíz duro seco en invierno del 2015. [1]

Para obtener un desgranado eficiente, el grado de humedad del maíz debe estar alrededor del $13 \%$ al $15 \%$ por lo que se debe someter a métodos de secado, uno de ellos es dejar la mazorca en la planta y exponerlo a las corrientes de aire a la temperatura ambiente [2].

Existen varias formas para realizar el desgranado, la más común es quitando los granos de la mazorca con los dedos lo que demanda gran cantidad de tiempo. Otro método empleado, pero que ocasiona problemas de salud en la espalda, es frotar la mazorca sobre una tabla de madera provista de grapas, hasta que los granos se desprendan del núcleo tal como se observa en la Figura 2.

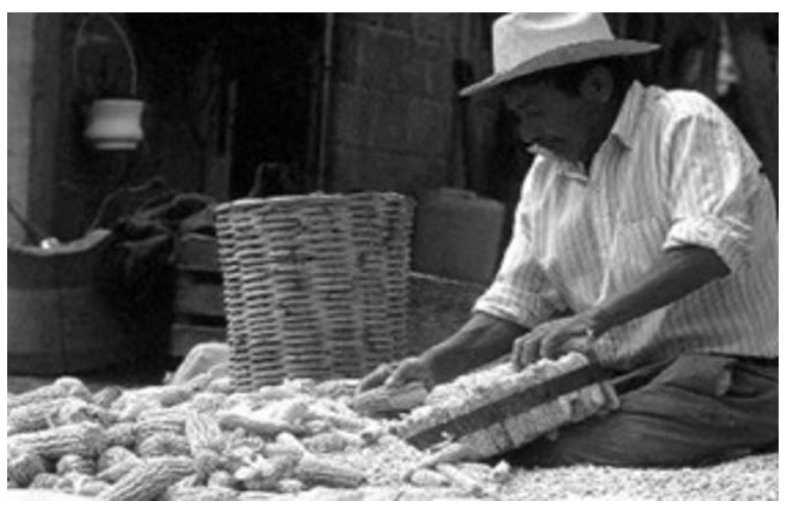

Figura 2. Desgranado mediante tabla con grapas. [3]
Debido al aumento de la demanda, los pequeños agricultores han visto la necesidad de tomar medidas que les permitan incrementar su producción en menor tiempo, razón por la cual se plantea el diseño y fabricación de una desgranadora de maíz para ayudar a los pequeños agricultores y mitigar lesiones producidas por el proceso manual.

La máquina realizada en Autodesk Inventor Professional se presenta en la Figura 3.

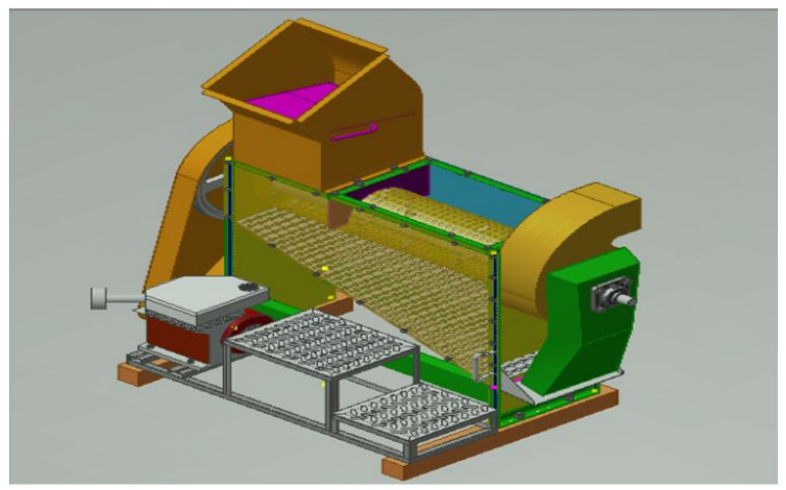

Figura 3. Máquina para desgranar maíz.

\section{Diseño y construcción}

El diseño de la máquina inicia con el cálculo de la producción requerida de aproximadamente 15 quintales/hora; cada quintal de maíz contiene alrededor de 125 mazorcas que dependerán de su tamaño [4].

\subsection{Dados desgranadores}

Como se observa en la Figura 4, los dados en la máquina cumplen la función de un dedo pulgar que en el desgranado manual se utiliza para arrancar varios granos de su núcleo. Por esta razón el dado tiene un diámetro de $19 \mathrm{~mm}$ y una longitud de $60 \mathrm{~mm}$ asemejando las dimensiones de un dedo pulgar promedio.

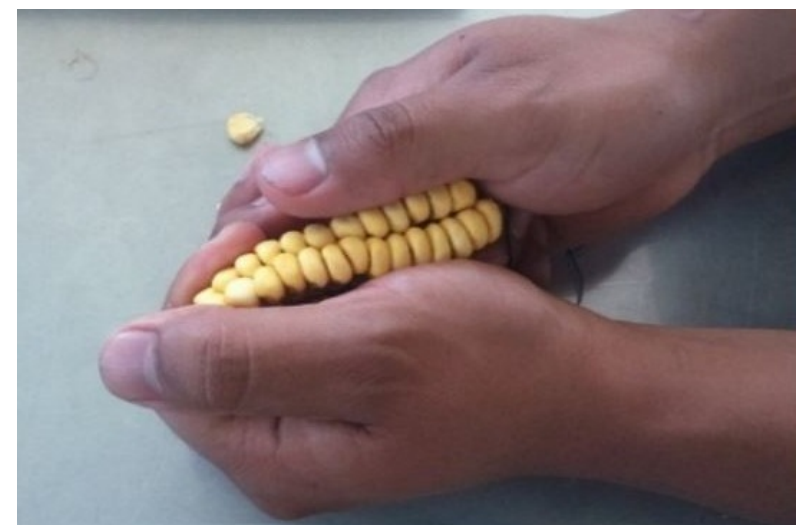

Figura 4. Sistema de desgranado manual.

Mediante ensayos en un dinamómetro de veinte mazorcas se determinó que para arrancar un promedio 
de 35 a 45 granos del núcleo se emplea aproximadamente $30 \mathrm{~N}$, además se debe tomar en cuenta que en las primeras hileras a desgranarse esta fuerza llega a aumentarse hasta un $50 \%$. En la Figura 5 se detalla el diagrama de cuerpo libre y se observa la distribución de la fuerza a lo largo del dado.

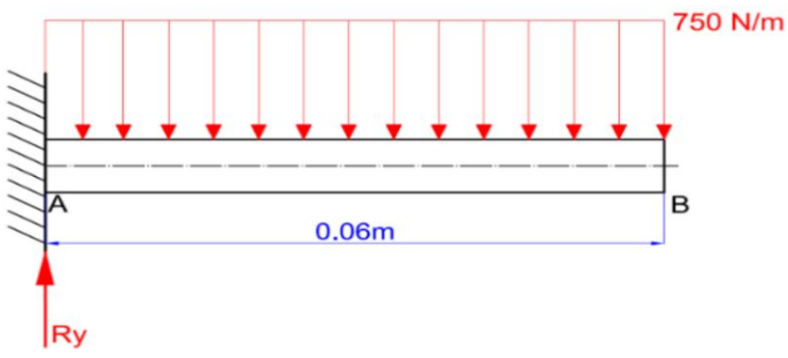

Figura 5. DCL dado desgranador.

Los principales esfuerzos a los cuales estará sometido el dado durante la operación de la máquina serán a flexión y a cortante; la magnitud de estos esfuerzos podrá determinarse al aplicar las ecuaciones 1 y 2 [5] respectivamente.

$$
\begin{gathered}
\sigma=\frac{32 M}{\pi d^{3}} \\
\tau=\frac{4 V}{3 a A}
\end{gathered}
$$

Donde:

$\sigma:$ esfuerzo de flexión

$\mathrm{M}$ : momento que interviene en el dado

d: diámetro del dado

$\tau$ : esfuerzo cortante

$\mathrm{V}$ : fuerza de corte en el lado

A: Área del dado

Para el diseño del dado desgranador el material empleado será SAE 1018 [6] porque posee buena soldabilidad, además, su maquinado es mejor y se emplea para ejes de transmisión con moderada exigencia al torque.

Realizado el cálculo de esfuerzos se procede con el análisis del dado, con el módulo de esfuerzo (stress) que Autodesk Inventor Professional cuenta entre sus opciones, incluyendo cálculo de tensiones, deformación y coeficiente de seguridad.

La fuerza de arranque de los granos de maíz también depende de la humedad del producto [2] y la capacidad a producir, por esa razón la fuerza de arranque está en un rango de 30-50 N.

En la Figura 6 se detalla la simulación del desplazamiento en la parte intermedia del dado, para determinar el desplazamiento y factor de seguridad. Se empleó la carga máxima de desgranado de $50 \mathrm{~N}$ en el dado.
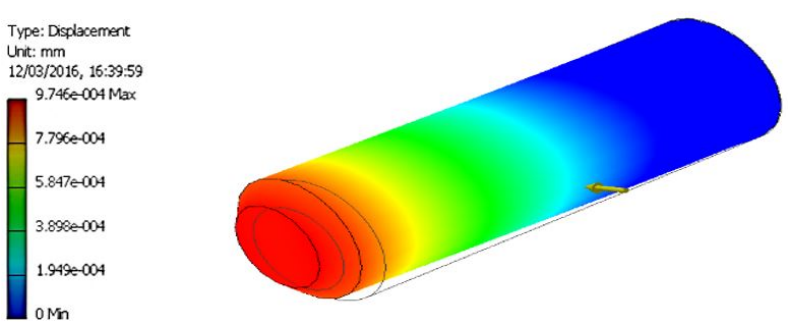

Figura 6. Simulación del dado.

Al realizar la simulación se obtienen datos los mismos que de forma detallada se encuentran en la Tabla 1.

Tabla 1. Análisis de la simulación del dado

\begin{tabular}{lcc}
\hline Name & Minimum & Maximum \\
\hline Volume & \multicolumn{2}{c}{$168603 \mathrm{~mm}^{3}$} \\
Mass & \multicolumn{2}{c}{$0.132691 \mathrm{~kg}$} \\
Weight & \multicolumn{2}{c}{$1.305 \mathrm{~N}$} \\
Von Mises & $0.00135716 \mathrm{MPa}$ & $1.76423 \mathrm{MPa}$ \\
1st Principal & $-1.10062 \mathrm{MPa}$ & $2.82418 \mathrm{MPa}$ \\
Stress & & \\
3rd Principal & $-2.83854 \mathrm{MPa}$ & $1.08788 \mathrm{MPa}$ \\
Stress & $0 \mathrm{~mm}$ & $0.000974562 \mathrm{~mm}$ \\
Displacement & $3 \mathrm{ul}$ & $15 \mathrm{ul}$ \\
Safety Factor & & \\
\hline
\end{tabular}

\subsection{Cilindro desgranador}

El cilindro como se observa en la Figura 7, es el elemento que cumple la función de contener todos los dados, los cuales estarán soldados.

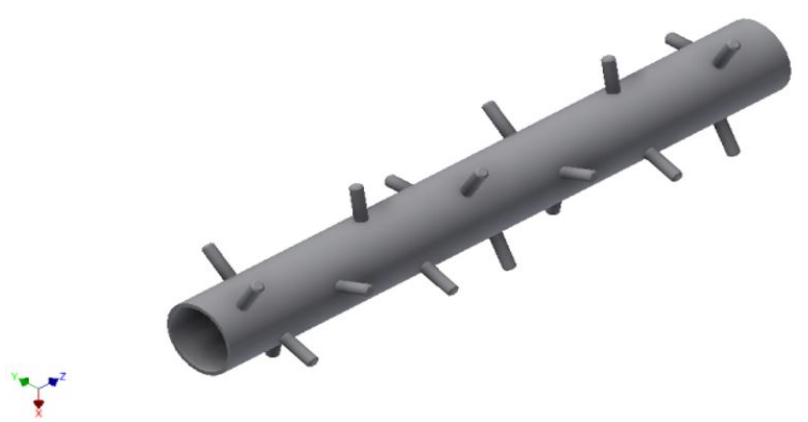

Figura 7. Cilindro desgranador

Los dados se ubican en el cilindro siguiendo un recorrido en espiral a todo su alrededor tal como se detalla en la Figura 8. Para proporcionar un rápido desgrane y transporte de expulsión de su núcleo, se colocan en tres hileras para no producir atascamientos ni roturas de la mazorca [2]. 

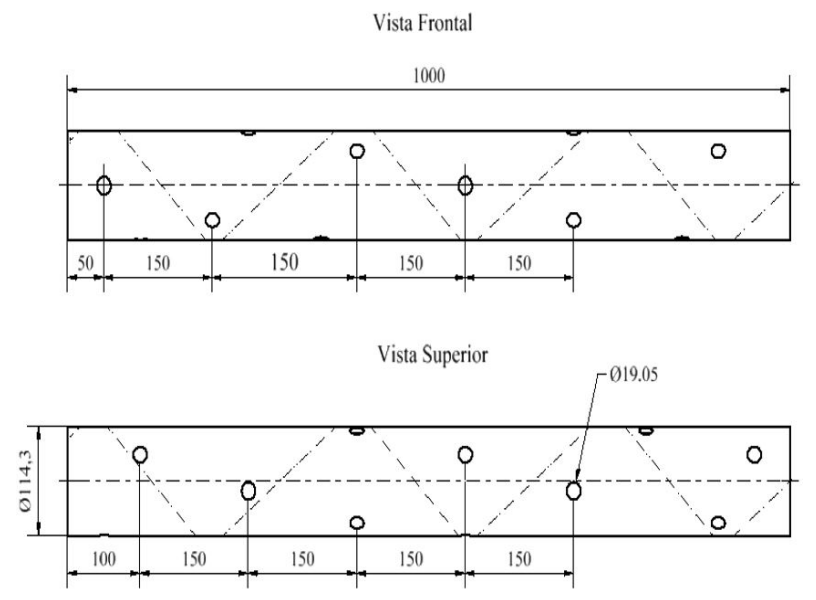

Figura 8. Recorrido en espiral.

Mediante experimentos y criterios de ingeniería se propuso que la distancia entre dados debe ser el promedio de tamaño y diámetro de mazorcas. Las dimensiones de las mismas de acuerdo con su tamaño fueron determinadas en la Tabla 2.

Tabla 2. Dimensiones de las mazorcas de maíz

\begin{tabular}{lcc}
\hline \multicolumn{1}{c}{ Tipo } & Diámetro $(\mathbf{m m})$ & Tamaño $(\mathbf{m m})$ \\
\hline Pequeño & $\geq 43,48$ & $\geq 10,94$ \\
Mediano & $\geq 49,4$ & $\geq 14,92$ \\
Grande & $\geq 53,04$ & $\geq 17,22$ \\
\hline
\end{tabular}

Para la colocación de los dados a lo largo del cilindro se realiza una distribución equitativa en cada hilera y se realiza con la siguiente ecuación 3:

$$
\begin{gathered}
\text { \#dados }=\frac{l}{d} \\
\# \text { dados }=\frac{1 \mathrm{~m}}{0.15 \mathrm{~m}} \\
\# \text { dados }=6.66 \approx 7
\end{gathered}
$$

\subsection{Diseño de la criba}

La criba o tamiz que se observa en la Figura 9, es la cámara perforada que recubre en su totalidad al cilindro desgranador que cumple la función de prelimpiado del maíz desgranado, mientras su núcleo, polvo y partículas más livianas salen expulsadas por el ventilador.

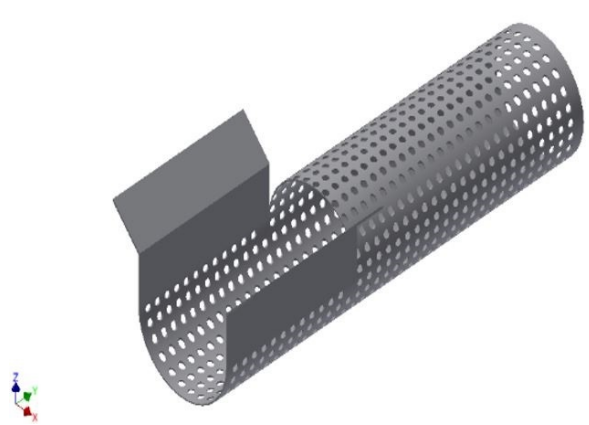

Figura 9. Criba

El diseño de la criba está en función del volumen de producción, tamaño y diversas formas físicas de granos de maíz, además del amplio camino de los núcleos hacia el ventilador, los datos de diseño se obtuvieron experimentalmente, así para un volumen aproximado de producción de 15 q se ocupa un cilindro de 250 $\mathrm{mm}$ de diámetro, cumpliendo con los factores antes mencionados.

El diámetro del cilindro desgranador se obtiene mediante la comparación entre el diámetro de la criba y la distancia de los dados desgranadores calculados previamente (Figura 10). Los dados y la criba deben estar separados para no producir atascamientos.

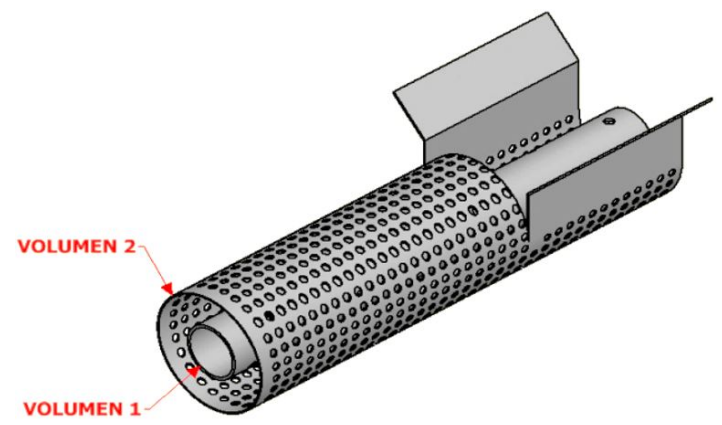

Figura 10. Comparación de volúmenes entre criba y cilindro desgranador.

En la Tabla 3 se determina experimentalmente el valor promedio del volumen que ocupan 125 mazorcas en un quintal de maíz.

Tabla 3. Dimensiones de las mazorcas de maíz

\begin{tabular}{cc}
\hline Experimento & $\begin{array}{c}\text { Volumen de 125 } \\
\text { mazorcas }\left(\mathbf{m}^{3}\right)\end{array}$ \\
\hline 1 & 0,04123 \\
2 & 0,04154 \\
3 & 0,04233 \\
4 & 0,04275 \\
5 & 0,04103 \\
Promedio & $\mathbf{0 , 0 4 1 7 7 6}$ \\
\hline
\end{tabular}


Mediante la ecuación 4 y los datos de volumen de la criba y de las mazorcas que se tomaron experimentalmente se determina el diámetro del cilindro.

$$
\begin{gathered}
V_{\text {mazorcas }}=V_{\text {criba }}-V_{\text {cilindro }} \\
0,0417776 \mathrm{~m}^{3}=\left[\pi \times(0,125 \mathrm{~m})^{2} \times 1 \mathrm{~m}\right]- \\
-\left[\pi \times\left(\text { radio }_{\text {cilindro }}\right)^{2} \times 1 \mathrm{~m}\right] \\
\text { Diámetro } \\
\text { cilindro }=3,78 \mathrm{plg} \approx 4 \mathrm{plg}
\end{gathered}
$$

\subsection{Diseño del eje principal}

Para un diseño correcto se consideran los elementos que van a soportar el eje, tales como la polea, el ventilador y el cilindro desgranador como se observan en la Figura 11.

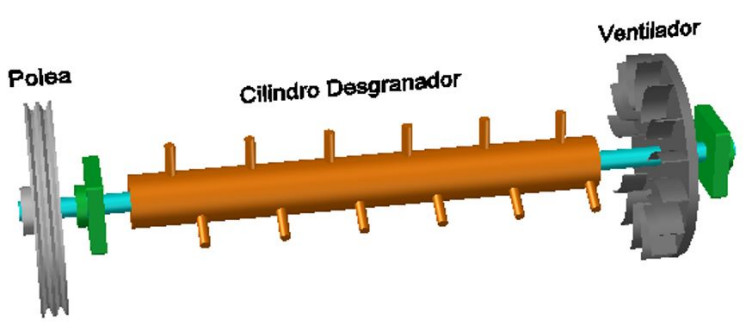

Figura 11. Elementos que soporta el eje.

El material empleado es acero AISI 4140, [6] bonificado de alta resistencia a la tracción y torsión, además, es recomendado para la construcción de ejes. En la Tabla 4 se indican los datos técnicos.

Tabla 4. Propiedades mecánicas del acero AISI 4140 [6]

\begin{tabular}{ccccc}
\hline $\begin{array}{c}\text { Diámetro } \\
\mathbf{m m}\end{array}$ & $\begin{array}{c}\text { Límite de } \\
\text { fluencia } \\
\mathbf{N} / \mathbf{m m}^{2}\end{array}$ & $\begin{array}{c}\text { Resistencia a } \\
\text { la tracción } \\
\mathbf{N} / \mathbf{m m}^{2}\end{array}$ & $\begin{array}{c}\text { Elongación } \\
\mathbf{N} / \mathbf{m m}^{2}\end{array}$ & $\begin{array}{c}\text { Reducción de } \\
\text { área } \mathbf{N} / \mathbf{m m}^{2}\end{array}$ \\
\hline$\leq 16$ & 900 & $1100-1300$ & 10 & 40 \\
$\leq 16 \leq 40$ & 750 & $1100-1200$ & 11 & 45 \\
$\leq 40 \leq 100$ & 650 & $900-1100$ & 12 & 50 \\
$\leq 100 \leq 160$ & 550 & $800-950$ & 13 & 60 \\
$\leq 160 \leq 250$ & 500 & $750-900$ & 14 & 65 \\
\hline
\end{tabular}

Para el diseño se toma en cuenta que el momento generado por la polea es igual al del ventilador para que se estabilicen las fuerzas. En la Figura 12 se observa el diagrama de cuerpo libre del eje.

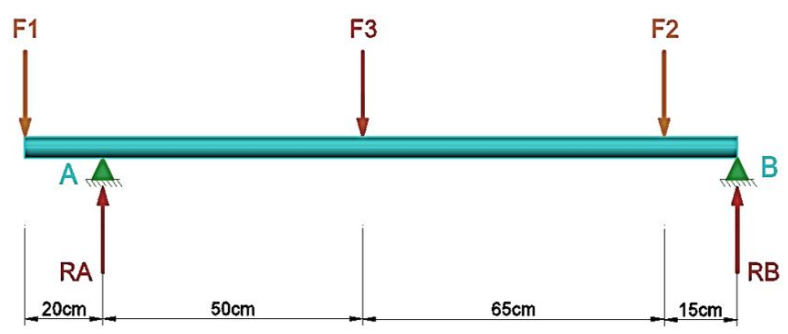

Figura 12. Diagrama de cuerpo libre del eje.
Los diagramas de fuerza cortante y de momentos, así como las reacciones en los soportes A y B, y el momento máximo se obtienen en el software MDSolids como se indica en la Figura 13.

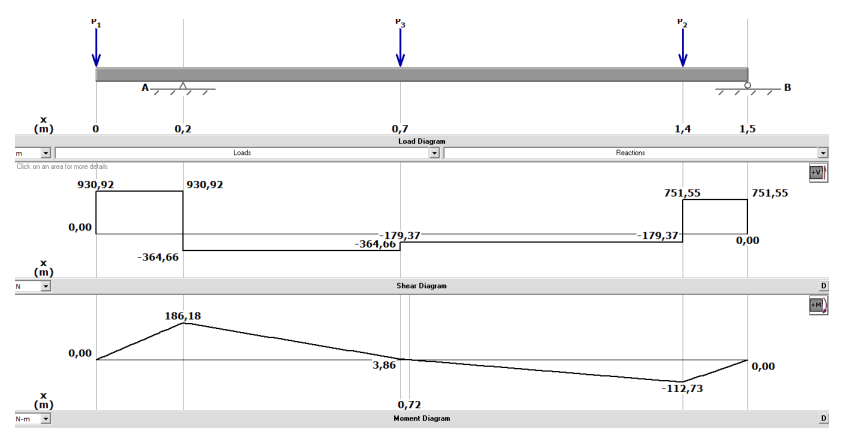

Figura 13. Diagrama de cargas, fuerza cortante y momentos.

El eje va a estar sometido a esfuerzos de flexión y torsión $(\tau)$ [5], y para determinar su diámetro se realiza primero un predimensionamiento mediante análisis estático. Se calcula el esfuerzo admisible $(\sigma)[5]$ y el diámetro (d) [5].

$$
\tau=\frac{16 T}{\pi d^{3}}
$$

$$
\sigma=\frac{S_{y}}{F_{s}}
$$

$$
d=\sqrt[6]{\frac{(32 M)^{2}+3(16 T)^{2}}{\pi\left(\sigma_{e q}^{2}\right)}}
$$

Para la optimización del diseño se realiza un estudio dinámico obteniendo el diámetro mediante el método de Goodman modificado [7].

$$
d=\sqrt[3]{\frac{48 F_{s}}{\pi} \sqrt{\left(\frac{T}{s_{y}}\right)^{2}+\left(\frac{M}{s_{e}}\right)^{2}}}
$$

\subsection{Diseño del ventilador}

Para expulsar las impurezas y núcleos de maíz, se instala un ventilador como se observa en la Figura 14, el cual se sitúa al final del sistema de desgranado. 

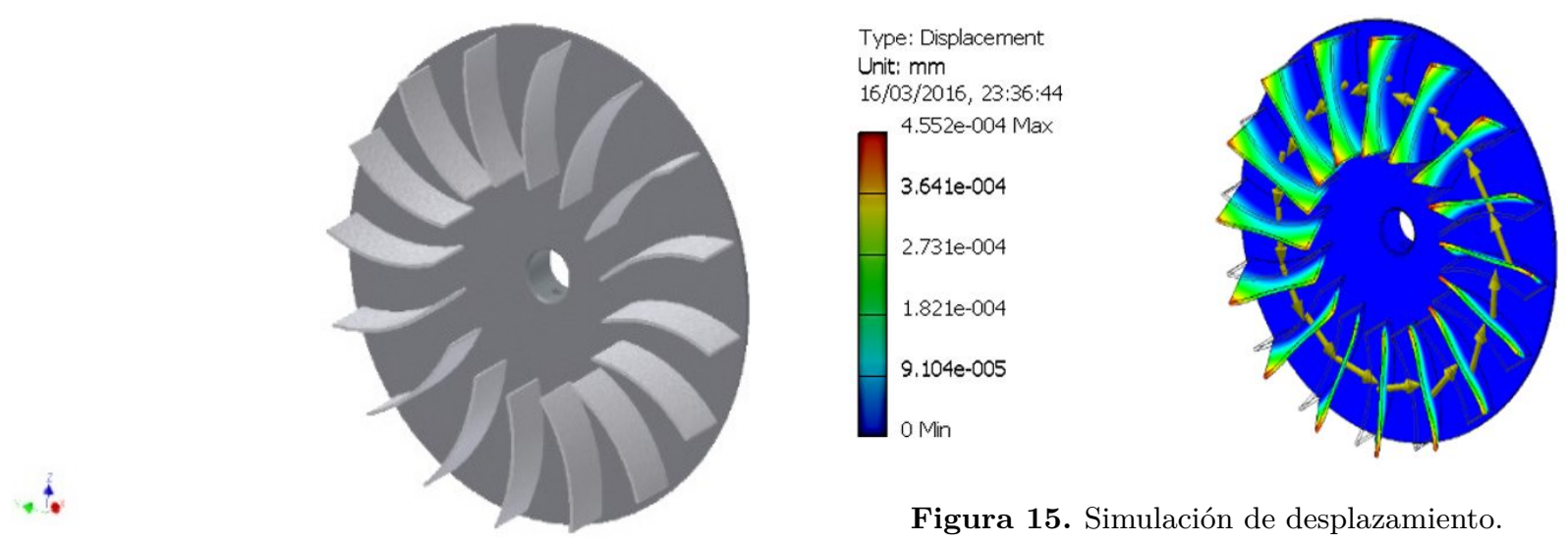

Figura 15. Simulación de desplazamiento.

Figura 14. Álabes del ventilador.

Los ventiladores centrífugos de aspas con curvatura hacia atrás son ideales en lo que se refiere a transportación y extracción; además, las aspas tienden a ser de autolimpieza y poseen una amplia capacidad de presión y volumen [8].

La parte fundamental en el diseño es determinar la velocidad necesaria para separar los núcleos e impurezas de los granos de maíz, empleando la ecuación 9.

$$
v=\sqrt{\frac{2 R}{c A_{P} \delta_{a}}}
$$

Donde las principales variables son:

v: velocidad ascendente de expulsión

R: resistencia que opone un cuerpo a moverse

c: coeficiente de arrastre adimensional

$\mathrm{A}_{\mathrm{p}}$ : área de proyección del cuerpo perpendicular a la velocidad

$\delta_{\mathrm{a}}$ : densidad absoluta del aire en el medio que se encuentra

La fuerza de la corriente de aire debe ser mayor al peso del núcleo (aproximadamente $0,085 \mathrm{~N}$ ) para que pueda expulsarla, caso contrario no se elevará.

La velocidad a la salida del ventilador $\left(v_{0}\right)$ se obtiene con la ecuación 10. Luego se procede al cálculo del caudal (Q).

$$
\begin{gathered}
\frac{v}{v_{0}}=0,86 \\
Q=v_{0} \times A_{b o c a} \text { de salida }
\end{gathered}
$$

El ventilador consta de 16 álabes debido a que para ventiladores con curvatura hacia atrás el número de aspas debe ser de 12 a 16 [9].

Para realizar la comprobación del ventilador se realiza la simulación de los álabes tanto en desplazamiento como factor de seguridad para garantizar la durabilidad. En las Figuras 15 y 16 se observan la simulación de desplazamiento y factor de seguridad.

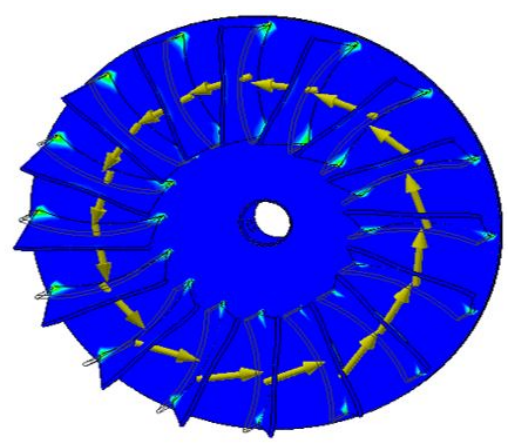

Figura 16. Simulación de factor de seguridad.

En la Figura 17 se observa la máquina ya construida.

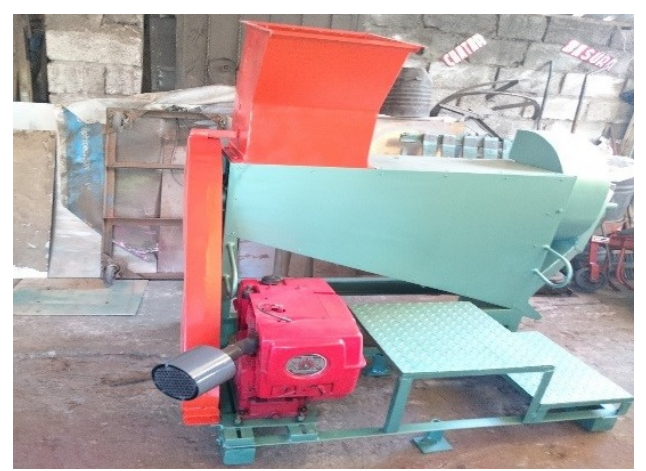

Figura 17. Desgranadora construida.

\section{Análisis experimental de la desgranadora}

Para el experimento se plantea la comprobación del funcionamiento correcto de la máquina y obtención del producto desgranado.

Se realiza en un laboratorio el análisis de humedad de diversos maíces a ser desgranados [10] para poder tabular y concluir qué maíz es el indicado a desgranar sin obtención de pérdidas ni daños en el producto. En la Tabla 5 se observa los datos de humedad de cada tipo de maíz. 
Tabla 5. Grado de humedad de varias muestras de maíz

\begin{tabular}{ccc}
\hline Ítem & Tipo de maíz & $\begin{array}{c}\text { Porcentaje de } \\
\text { humedad }\end{array}$ \\
\hline Muestra 1 & Maíz harinoso & $11,98 \%$ \\
Muestra 2 & Maíz suave & $34,05 \%$ \\
Muestra 3 & Morocho & $10,18 \%$ \\
Muestra 4 & Maíz dentado & $14,32 \%$ \\
\hline
\end{tabular}

1) Prueba 1 a 800 rpm: se logró determinar el tiempo que demora en desgranar como la fisonomía del desgranado de cada tipo de muestra, como se observa en la Tabla 6 .

Tabla 6. Tabulación prueba 1

\begin{tabular}{cccc}
\hline Ítem & $\begin{array}{c}\text { Tiempo de } \\
\text { desgranado } \\
(\text { min) }\end{array}$ & $\begin{array}{c}\text { Cantidad de } \\
\text { maíz } \\
\text { desgranado (q) }\end{array}$ & $\begin{array}{c}\text { Aspecto de } \\
\text { desgranado } \\
\text { (visual) }\end{array}$ \\
\hline Muestra 1 & 0,56 & $1 / 4$ & Entero \\
Muestra 2 & 0,94 & $1 / 4$ & Destrozado \\
Muestra 3 & 0,4 & $1 / 4$ & Entero \\
Muestra 4 & 0,43 & $1 / 4$ & Entero \\
\hline
\end{tabular}

Se realiza una ponderación como se observa en la Figura 18, con cada muestra respecto al tiempo de desgranado.

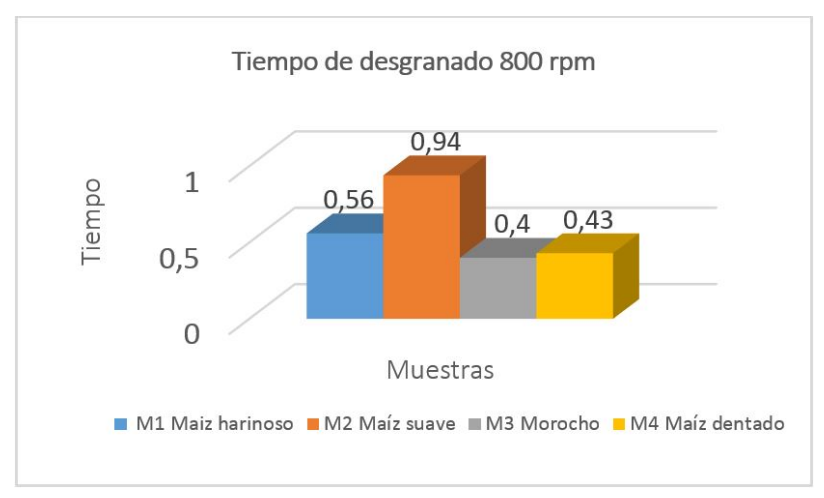

Figura 18. Ponderación de prueba 1.

Como análisis de la primera prueba, se obtiene que el maíz con grado de humedad mayor se demora más tiempo en desgranar; además, su aspecto es quebradizo. En el caso contrario el maíz con menor grado de humedad desgrana más rápido y el grano se obtiene entero.

2) Prueba 2 a 1000 rpm: se logró determinar el tiempo que demora en desgranar como la fisonomía del desgranado de cada tipo de muestra, como se observa en la Tabla 7.
Tabla 7. Tabulación prueba 2

\begin{tabular}{cccc}
\hline Ítem & $\begin{array}{c}\text { Tiempo de } \\
\text { desgranado } \\
(\text { min) }\end{array}$ & $\begin{array}{c}\text { Cantidad de } \\
\text { maíz } \\
\text { desgranado (q) }\end{array}$ & $\begin{array}{c}\text { Aspecto de } \\
\text { desgranado } \\
\text { (visual) }\end{array}$ \\
\hline Muestra 1 & 0,43 & $1 / 4$ & Semientero \\
Muestra 2 & 0,81 & $1 / 4$ & Destrozado \\
Muestra 3 & 0,32 & $1 / 4$ & Entero \\
Muestra 4 & 0,37 & $1 / 4$ & Semientero \\
\hline
\end{tabular}

Se realiza una ponderación, como se observa en la Figura 19, con cada muestra respecto al tiempo de desgranado.

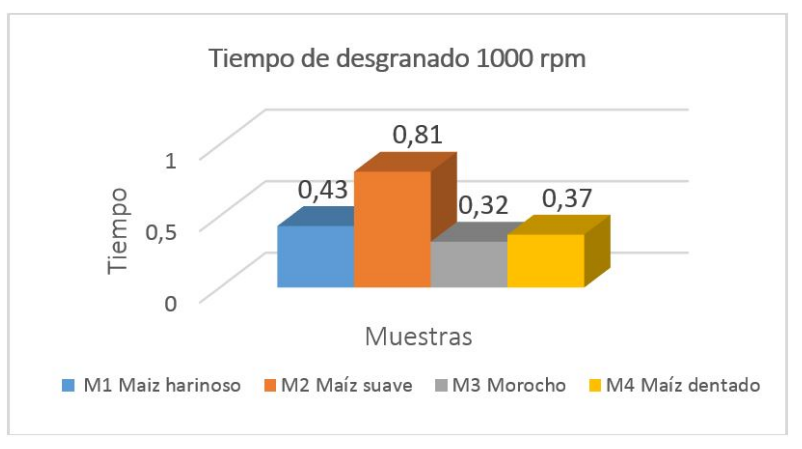

Figura 19. Ponderación de prueba 2.

Como resultado se observa que al aumentar las revoluciones el maíz se desgrana más rápidamente, pero algunos granos resultan quebradizos.

3) Prueba 3 a varios tamaños: se verifica si el tamaño de la mazorca influye en la producción de la máquina, como se observa en la tabla 8.

Tabla 8. Tabulación prueba 3

\begin{tabular}{cccc}
\hline Ítem & Especificaciones & $\begin{array}{c}\text { Cantidad de } \\
\text { maíz } \\
\text { desgranado (q) }\end{array}$ & $\begin{array}{c}\text { Aspecto de } \\
\text { desgranado } \\
\text { (visual) }\end{array}$ \\
\hline Prueba 1 & $\begin{array}{c}\text { Tamaños } \\
\text { pequeños } \\
\text { Diversos } \\
\text { tamaños } \\
\text { Prueba 2 }\end{array}$ & $1 / 4$ & Semientero \\
Prueba 3 & grandes & $1 / 4$ & Desgranado \\
\hline
\end{tabular}

Al término del análisis, en la prueba 1 los tamaños pequeños de maíz no desgranan de forma correcta, obteniendo lo contrario en las pruebas 2 y 3 respectivamente, y que se logra visualizar en la figura 20 .

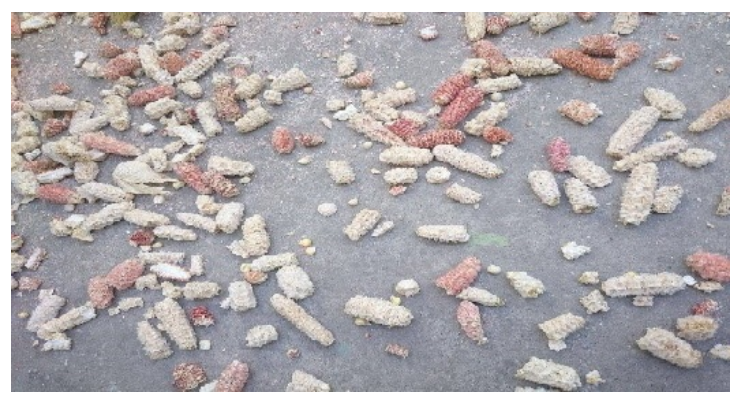

Figura 20. Maiz desgranado en su totalidad. 
4) Prueba 4 tiempos de desgranado: se logra determinar el tiempo de desgranado manual frente al desgranado mecánico, como se observa en la Tabla 9.

Tabla 9. Tabulación prueba 3

\begin{tabular}{cccc}
\hline Ítem & Especificaciones & $\begin{array}{c}\text { Cantidad de } \\
\text { maíz } \\
\text { desgranado (q) }\end{array}$ & $\begin{array}{c}\text { Tiempo de } \\
\text { desgranado } \\
\text { (minutos) }\end{array}$ \\
\hline Prueba 1 & $\begin{array}{c}\text { Desgranado } \\
\text { manual }\end{array}$ & $1 / 2$ & 25 \\
Prueba 2 & $\begin{array}{c}\text { Desgranado } \\
\text { máquina }\end{array}$ & $1 / 2$ & 0,54 \\
\hline
\end{tabular}

Se realiza una ponderación, como se observa en la Figura 21, con cada tipo de desgranado.

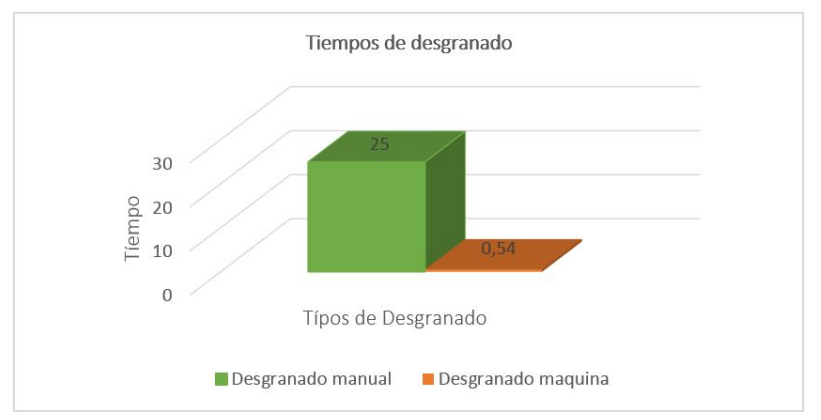

Figura 21. Ponderación de prueba 4.

$\mathrm{Al}$ término del análisis, en la prueba 1 el tiempo de desgranado es mayor, mientras en la prueba 2 es menor, obteniendo mayor volumen de producción.

5) Costo-beneficio: Para determinar el beneficio de la máquina, se realiza una comparación de producción de maíz desgranado manual vs. máquina. El desgranado manual produce alrededor de 10 q mientras la máquina produce aproximadamente 105 q en un día como se indica en la Figura 22; considerando el costo de una persona por día es alrededor de $\$ 20$ produciendo $10 \mathrm{q}$, mientras el costo máquina diario es de $\$ 35$ produciendo 105 q como se observa en la Figura 23.

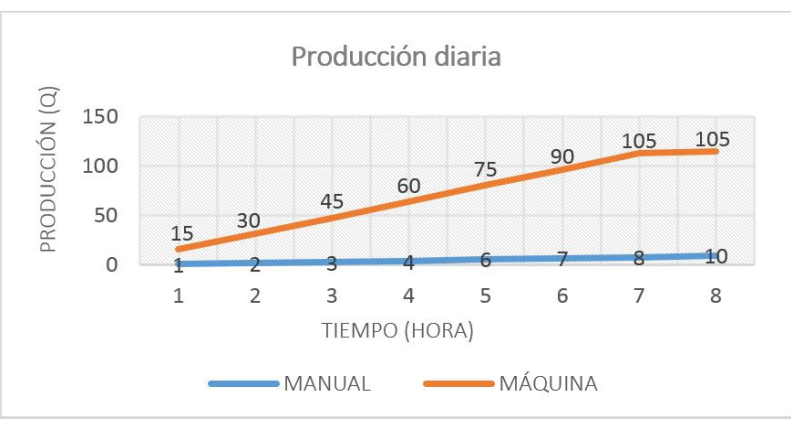

Figura 22. Producción diaria de maíz desgranado.

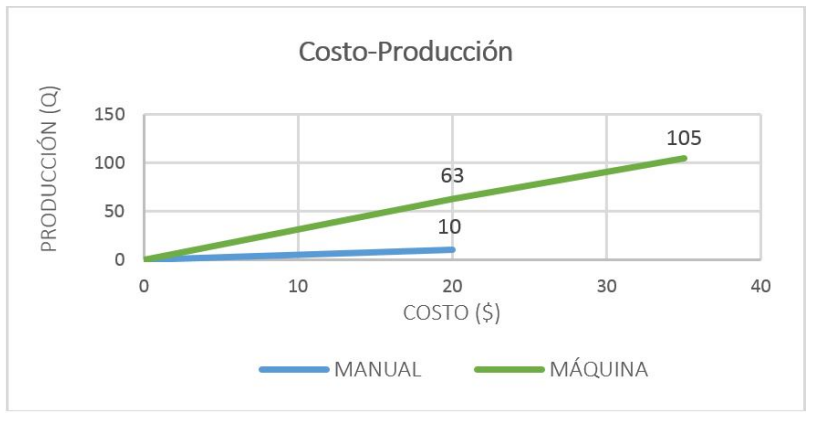

Figura 23. Costo-producción.

Como resultado se obtiene que desgranar manualmente 1 q de maíz cuesta USD2 mientras a máquina USD 0,33 .

6) VAN y TIR: para realizar el análisis del siguiente proyecto, se procede a calcular previamente los datos de egresos e ingresos del desgranado de maíz.

El rendimiento de maíz es de 190 q de 125 unidades/ha y una producción comercial en grano seco de 85 q/ha [2]. En la Tabla 10 se presenta la tabulación de costos.

Tabla 10. Tabulación de costos

\begin{tabular}{lccc}
\hline \multicolumn{1}{c}{ Indicador } & Diario & Mensual & Anual \\
\hline $\begin{array}{l}\text { Costo máquina } \\
\text { Costo hombre }\end{array}$ & $\$ 18,30$ & $\$ 366,00$ & $\$ 366,00$ \\
$\begin{array}{l}\text { Costo combustible } \\
\text { Mantenimiento }\end{array}$ & $\$ 16,86$ & $\$ 337,20$ & $\$ 337,20$ \\
$\begin{array}{l}\text { Producción } \\
\text { desgranado } \\
(\mathbf{8 5} \text { q/ha) }\end{array}$ & $\$ 135,15$ & $\$ 2.703,00$ & $\$ 2.703,00$ \\
\hline
\end{tabular}

Se debe tener en cuenta que la cosecha de maíz se da dos veces al año, con un período de 15 días de desgranado en una extensión de 2 hectáreas.

La tasa de rendimiento de la máquina es del $20 \%$, a continuación en la Tabla 11 se observa los cálculos realizados en tablas de Excel.

Tabla 11. Tabla de cálculo

\begin{tabular}{cccccc}
\hline Año & Ingreso & Egreso & Valor neto & $\begin{array}{c}\text { Valor } \\
\text { presente }\end{array}$ & FR \\
\hline $\mathbf{0}$ & $\$-$ & $\$ 2.000,00$ & $(\$ 2.000,00)$ & $(\$ 2.000,00)$ & $(\$ 2.000,00)$ \\
$\mathbf{1}$ & $\$ 2.703,00$ & $\$ 871,80$ & $\$ 1.831,20$ & $\$ 1.526,00$ & $(\$ 168,80)$ \\
$\mathbf{2}$ & $\$ 2.700,00$ & $\$ 831,80$ & $\$ 1.868,20$ & $\$ 1.297,36$ & $\$ 1.699,40$ \\
$\mathbf{3}$ & $\$ 2.695,00$ & $\$ 840,00$ & $\$ 1.855,00$ & $\$ 1.073,50$ & $\$ 3.554,40$ \\
$\mathbf{4}$ & $\$ 2.705,00$ & $\$ 835,50$ & $\$ 1,869,50$ & $\$ 901,57$ & $\$ 5.423,90$ \\
$\mathbf{5}$ & $\$ 2.703,00$ & $\$ 833,50$ & $\$ 1,869,50$ & $\$ 751,31$ & $\$ 7.293,40$ \\
\hline
\end{tabular}

Los resultados determinan que la desgranadora es rentable a corto plazo con un fondo de retorno o payback de 2 años, como se observa en Tabla 12.

Tabla 12. Cálculo del payback

\begin{tabular}{cccc}
\hline VAN & TIR & Payback & Asignación \\
$\$ 3549,74$ & $89 \%$ & 2 & Rentable \\
\hline
\end{tabular}




\section{Resultados}

Una vez realizado el análisis con los diversos tipos de maíces, se hallan los siguientes resultados:

Se determinó que el maíz por su grado de humedad tiene cambios en su proceso de desgranado, entre menor del $14 \%$ sea el grado de humedad más rápido será su producción en un $75 \%$.

$\mathrm{Al}$ introducir mazorcas de diversos tamaños, ver Tabla 8 , se observó que la pérdida en el desgranado es mínima, obteniendo una producción mayor, en casos especiales el maíz llega a salir casi entero o quebradizo cuando el tamaño es muy pequeño.

$\mathrm{Al}$ experimentar el desgranado se concluye que la máquina está diseñada y apta para trabajar con varios tipos de maíces con una humedad recomendada del 10 al $16 \%$.

A base de múltiples pruebas de funcionamiento, ver tablas 6 y 7 , se determinó que la velocidad en el cilindro es alrededor de $850 \mathrm{rpm}$, si la velocidad excede este valor, el grano tiende a romperse.

$\mathrm{Al}$ analizar la máquina respecto a un costo producción, ver Figura 23, resulta muy rentable, por motivos de tiempo de desgranado, mano de obra con relación a un desgranado manual, ver Figura 20 debido a que el costo diario desgranando manualmente es USD 2, mientras que con la máquina es USD 0,33.

$\mathrm{Al}$ realizar la comprobación del ventilador mediante simulación de sus álabes se obtuvieron resultados favorables tanto en desplazamiento como en el factor de seguridad que garantiza la funcionalidad y durabilidad.

Al realizar la simulación del dado desgranador se lograron resultados positivos tanto en factor de seguridad de $3 \mathrm{ul}$ como en desplazamiento de 0,001 mm, por lo que trabajará sin ningún problema.

En el análisis de VAN y TIR se determinó que la máquina es rentable a corto plazo con un fondo de retorno de 2 años, ver Tabla 12, ayudando al pequeño agricultor a tener grandes producciones en el menor tiempo, además de abastecer la demanda en el mercado y salvaguardando su salud física al evitar lesiones.

\section{Conclusiones}

El sistema de desgranado con dados se construyó para que el maíz no se rompa al momento de desgranar y así conseguir un producto entero, limpio y en el menor tiempo posible.

La máquina se diseñó de forma sencilla para que todos los materiales se puedan encontrar en el mercado nacional, lo que permite un fácil acceso a repuestos, y no afectar al pequeño agricultor en tiempo y dinero.

$\mathrm{Al}$ analizar las diferentes variables que pueden darse en la operación de la desgranadora, se determinó que el rendimiento de la máquina será mayor mientras el maíz se encuentre más seco y su grado de humedad no baje del $10 \%$.

En pruebas de funcionamiento la máquina tiene una capacidad de aproximadamente $17 \mathrm{q}$ /hora superior a la que se propuso en un principio, incrementando su producción, evitando así la fatiga laboral y cuidando la salud ocupacional de las personas.

\section{Referencias}

[1] A. Monteros and S. Salvador, "Rendimientos de maíz duro seco en el Ecuador invierno 2015." SINAGAP, 2015. [Online]. Available: https://goo.gl/gP8aZF

[2] J. Espin and J. Villavicencio, "Diseño y construcción de una máquina para el proceso de desgranado de maíz de la costa." in Tesis Ingeniero Mecánico. Escuela Superior Politécnica de Chimborazo, 2009. [Online]. Available: https://goo.gl/1PQ6Hd

[3] Maíces del Nansa. (2013) Las oloteras: Utensilios para desgranar maíz en México. [Online]. Available: https://goo.gl/oyBf7W

[4] INIAP. (2012) Variedad del maíz en Ecuador.

[5] R. Budynas and K. Nisbett, Diseño en ingeniería mecánica de Shigley, $9^{\text {th }}$ ed. McGraw-Hill Interamericana, 2012.

[6] Bhöler, "Catálogo de aceros," 2011. [Online]. Available: https://goo.gl/tMnZ5p

[7] E. Cuñas, "Diseño y construcción de un extractor centrífugo para almidón de yuca,." Universidad Politécnica Salesiana, 2014. [Online]. Available: https://goo.gl/wsn7sM

[8] R. Greene and F. Noriega, Compresores: selección, uso y mantenimiento. McGraw-Hill, 1987.

[9] PDVSA, "Manual de diseño de proceso: ventiladores," 2006.

[10] Universidad Central del Ecuador, "Informe de resultados," 2015. 